\title{
Extremsommer lässt baumförmige Aloen (Aloidendron) üppig im Palmengarten blühen
}

\author{
Christian WEHLMANN
}

\begin{abstract}
The sunny and hot extreme summer 2018 had a positive effect on the growth of succulent plants in the Palmengarten. Many species of the Palmengarten collection originate from the southern hemisphere and thus flower during the winter season, including many aloes. In the semi-desert and fog desert display of the Tropicarium, some of the arborescent aloes, Aloidendron ramosissimum and $A$. dichotomum, are cultivated. Both benefited from the extreme summer started to bloom in November 2018.
\end{abstract}

\section{Zusammenfassung}

Der sonnige und heiße Extremsommer 2018 hat sich positiv auf das Wachstum der Sukkulenten im Palmengarten ausgewirkt. Viele Arten der Palmengarten-Sammlung stammen von der Südhalbkugel und beginnen folglich bei uns im Winterhalbjahr, ihre Blüten zu öffnen, darunter auch viele Aloen. Im Halbwüsten- und dem Nebelwüsten-Haus des Tropicariums gedeihen einige Exemplare der baumförmigen Aloen Aloidendron ramosissimum und A. dichotomum. Sie haben von dem Extremsommer stark profitiert und reichlich Blüten angesetzt, die sich seit November 2018 kontinuierlich öffnen.

Der Sommer 2018 darf ohne Frage zu einem der hellsten, heißesten und trockensten Sommer seit der Jahrtausendwende gezählt werden. Mit durchschnittlich 790 Sonnenstunden in Hessen und einer Durchschnittstemperatur von $19,3{ }^{\circ} \mathrm{C}$ in Deutschland, kombiniert mit extrem wenig Nie-

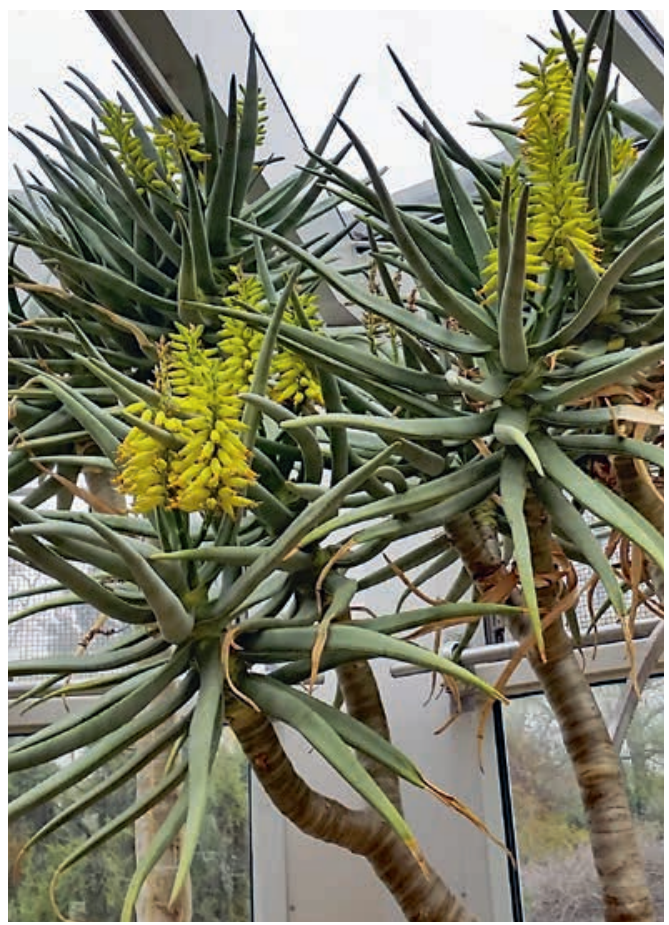

Abb. 1: Aloidendron dichotomum mit noch teilweise ungeöffneten Blüten. (Foto: C. Wehlmann) derschlag, hatten viele mitteleuropäische Pflanzen Probleme, sich auf diese wochenlange Extremwitterung einzustellen. Frei nach dem Spruch „des einen Freud ist des anderen Leid“ haben dagegen viele Pflanzen im Bereich „Trockene Tropen“ der Schauhäuser des Palmengartens dem Sommer mit reichem Wachstum und reger Blühfreudigkeit gedankt. Dank außergewöhnlich hoher Einstrahlung und fachgerechter gärtnerischer Pflege sind die positiven Effekte dieses Sommers bis jetzt zu bewundern. Im Herbst und Winter 2018 ist dies klar erkennbar an der ungewöhnlich früh und reichlich einsetzenden Blüte bei Arten der Gattungen Aloe und Aloidendron.

Die Gattung Aloidendron umfasst sieben Arten, wovon im Nebelwüstenhaus $A$. dichotomum (Köcherbaum) und $A$. ramosissimum gezeigt werden, die in Namibia und der südafrikanischen Provinz Nordkap vorkommen. Die Pflanzen unterscheiden sich durch ihre unterschiedliche Art der Verzweigung und sind im Nebelwüstenhaus in verschiedenen Altersstadien zu bewundern.

Beide bevorzugen ein mineralisches Substrat und sollten sparsam mit Wasser bedacht werden. Aus eigener Erfahrung reicht das Wässern der umliegenden Pflanzen vollkommen aus, sodass die gut eingewurzelten Pflanzen das ganze Jahr praktisch trocken stehen. Bei ausgewachsenen Pflanzen geht der Blühbeginn mit dem afrikanischen Frühling 


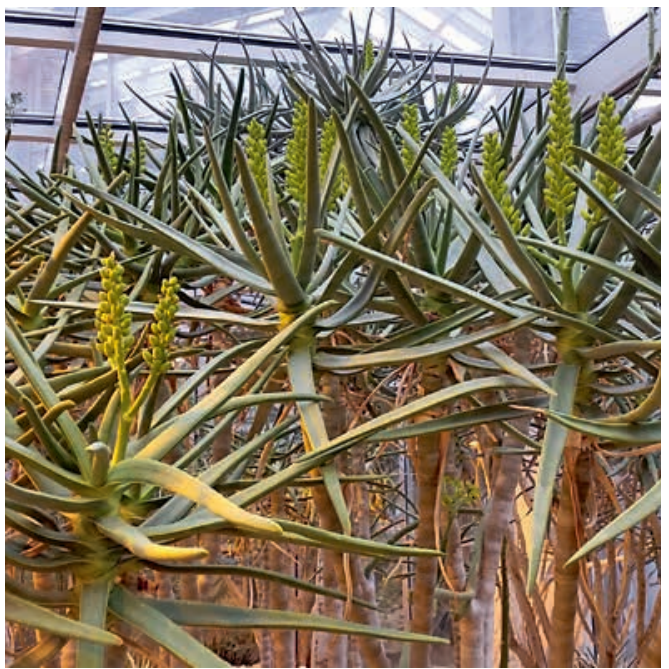

Abb. 2: Aloidendron ramosissimum mit Blütenknospen in der Nebelwüste. (Foto: C. Wehlmann)

einher, der jahreszeitlich gesehen in Europa leider den Winter einläutet. Zur Unterstützung der Pflanzen werden daher zusätzliche Leuchtmittel eingesetzt, um genügend Helligkeit für eine ausreichende Entwicklung der Blüten zu garantieren. Angetrieben durch den für die Pflanzen sehr wertvollen Sommer 2018 öffneten sich die ersten Knospen etwa seit Ende September 2018.

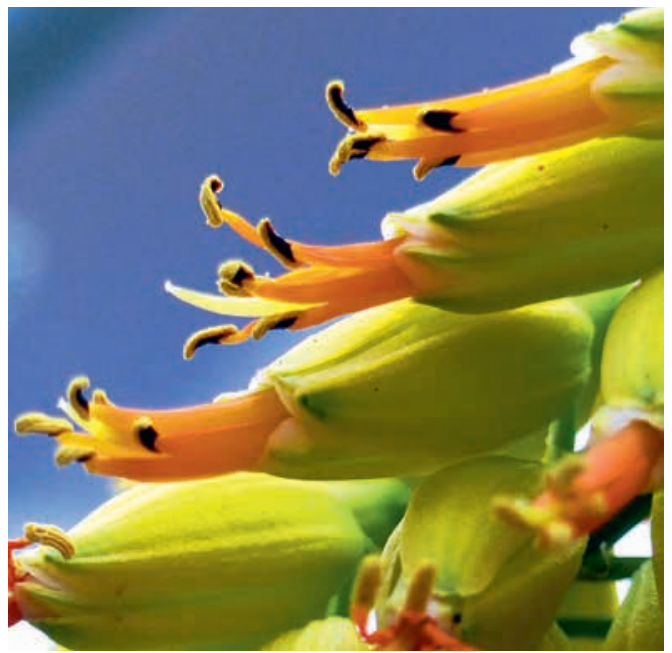

Abb. 4: Drei geöffnete Blüten mit leuchtend orange gefärbten Filamenten aus einem Blütenstand von Aloidendron dichotomum. (Foto: H. STEINECKe)

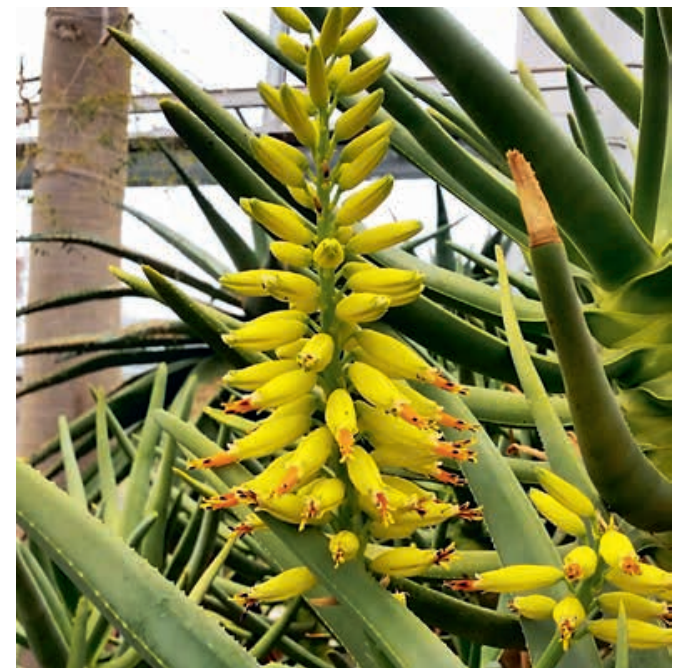

Abb. 3: Erste geöffnete Blüten von Aloidendron dichotomum. (Foto: C. WehlmanN)

Nicht nur in der Natur, sondern auch im Gewächshaus müssen die Aloidendron-Pflanzen einigen Widrigkeiten trotzen. So besteht immer die Gefahr eines Pilzeintrags über die Wurzel oder mögliche Verletzungen. Um dies auszuschließen, wird bei Bedarf mit zugelassenen Fungiziden oder präventiv mit Nutzpilzen gearbeitet, welche für die Pflanze schädliche Pilze fernhalten sollen. Auch ein Befall mit Wollläusen ist möglich. Diese werden jedoch durch mechanisches Abspritzen der Pflanze mit Wasser und dem Einsatz des australischen Marienkäfers im Zaum gehalten.

Die Pflanzen sind im Nebelwüstenhaus nur während der Öffnungszeiten (montags zwischen 9 und 14 Uhr) zu besichtigen, für weitere Zeiten können Sie bei der Grünen Schule des Palmengartens auch besondere Führungen durch dieses Haus buchen. Weitere Exemplare von Aloidendron dichotomum und $A$. ramosissimum sind auch im Halbwüstenhaus zu den Öffnungszeiten des Tropicariums zu sehen.

\section{Anschrift des Autors}

Christian Wehlmann, Palmengarten Frankfurt, Siesmayerstraße 61, 60323 Frankfurt, E-Mail: christian.wehlmann@ stadt-frankfurt.de 